\title{
The fission yeast meiosis-specific Dmc1 recombinase mediates formation and branch migration of Holliday junctions by preferentially promoting strand exchange in a direction opposite to that of $\operatorname{Rad} 51$
}

\author{
Yasuto Murayama, ${ }^{1}$ Yasuhiro Tsutsui, and Hiroshi Iwasaki ${ }^{2}$ \\ Department of Life Science, School and Graduate School of Bioscience and Biotechnology, Tokyo Institute of Technology, \\ Kanagawa 226-8501, Japan
}

Homologous recombination proceeds via the formation of several intermediates including Holliday junctions (HJs), which are important for creating crossover products. DNA strand exchange is a core reaction that produces these intermediates that is directly catalyzed by RecA family recombinases, of which there are two types in eukaryotes: universal Rad51 and meiosis-specific Dmc1. We demonstrated previously that Rad51 promotes four-strand exchange, mimicking the formation and branch migration of HJs. Here we show that Dmc1 from fission yeast has a similar activity, which requires ATP hydrolysis and is independent of an absolute requirement for the Swi5-Sfr1 complex. These features are critically different from three-strand exchange mediated by Dmc1, but similar to those of four-strand exchange mediated by Rad51, suggesting that strand exchange reactions between duplex-duplex and single-duplex DNAs are mechanistically different. Interestingly, despite similarities in protein structure and in reaction features, the preferential polarities of Dmc1 and Rad51 strand exchange are different (Dmc1 promotes exchange in the $5^{\prime}-$-to-3' direction and Rad51 promotes exchange in the $3^{\prime}$-to-5' direction relative to the ssDNA region of the DNA substrate). The significance of the Dmc1 polarity is discussed within the context of the necessity for crossover production.

[Keywords: homologous recombination; Holliday junction; recombinase; branch migration; Dmc1; meiosis]

Supplemental material is available for this article.

Received September 28, 2010; revised version accepted January 10, 2011.

Generally, homologous recombination $(\mathrm{HR})$ is initiated at DNA double-strand breaks (DSBs), which are subsequently converted by enzymatic degradation into resected DNA duplexes with 3'-overhanging ssDNA tails (Sun et al. 1991; Mimitou and Symington 2009). In one of several proposed HR pathways, the ssDNA end invades an intact homologous dsDNA, forming a D-loop structure. The D-loop is extended by DNA synthesis using the invading 3' DNA end as a replication primer. Subsequently, the opposite DSB end is captured by the displaced ssDNA derived from the initial strand invasion, and two strand exchange reactions at the ends of the D-loop proceed further, resulting in the formation of a double Holliday junction $(\mathrm{dHJ})$ intermediate. Resolution of the $\mathrm{dHJ}$ in one

\footnotetext{
${ }^{1}$ Present address: Cancer Research UK, London Research Institute, 44 Lincoln's Inn Fields, London, WC2A 3LY, UK.

${ }^{2}$ Corresponding author.

E-MAIL hiwasaki@bio.titech.ac.jp; FAX 81-45-924-5735.

Article is online at http://www.genesdev.org/cgi/doi/10.1101/gad.1997511.
}

orientation was predicted to yield crossover $(\mathrm{CO})$ recombinants, whereas resolution in the other orientation resulted in noncrossover (NCO) recombinants. This is referred to as the DNA DSB repair (DSBR) model, as originally proposed by Szostak et al. (1983). However, later studies suggested that the DSBR pathway is programmed to produce predominantly COs, and that NCO recombinants are produced by an alternative pathway referred to as synthesisdependent strand annealing (SDSA) (Allers and Lichten 2001; Hunter and Kleckner 2001). In the SDSA pathway, NCOs are produced by displacement of the extended invading strand into a D-loop, followed by reannealing to the $3^{\prime}$-tailed ssDNA on the other side of the break. Thereby, the production of COs, which play a very important role in meiotic segregation, must involve $\mathrm{HJ}$ intermediates formed only during the DSBR pathway, whereas NCOs can be produced without HJ formation.

In both pathways, DNA strand exchange is the central reaction and is promoted by evolutionarily conserved RecA family recombinases, including RecA in bacteria, 
Rad51 and Dmc1 in eukaryotes, and RadA in archaea. These recombinases are loaded onto ssDNA tails, where they form a helical filament, which is a substantial molecular structure for strand exchange (Sung and Klein 2006; Cox 2007). It has been shown that, irrespective of the recombinase involved, strand exchange occurs in vitro in an ATP-dependent manner (Bianco et al. 1998; Galkin et al. 2006; Cox 2007). The overall structures of the filaments created by the four groups of recombinases are indistinguishable by electron microscopy (Yang et al. 2001; Yu et al. 2001; Sehorn et al. 2004), indicating an evolutionary conservation of the fundamental reaction mechanism in all organisms.

Recombinase-mediated strand exchanges are often analyzed by three kinds of in vitro assays (Supplemental Fig. S1). These reactions are considered to mimic the early steps of the in vivo reactions, including D-loop formation and concomitant strand exchange. The fundamental mechanism of the strand exchange can be analyzed by a three-strand exchange, in which two homologous DNA molecules of circular ssDNA (css) and linearized dsDNA (ldsDNA) are used as substrates. After the formation of D-loops, HJs are formed, at least in principle, when continuing strand exchange proceeds past the original ss-ds junction of the invading DNA. This reaction beyond the ss and ds junction is closely related to the branch migration (BM) of HJs, which includes duplex-duplex reciprocal strand exchange or four-strand exchange. More than 20 years ago, RecA was shown in several assays to promote the formation and $\mathrm{BM}$ of $\mathrm{HJs}$, including in a four-strand exchange between a gapped circular DNA (gDNA) and its homologous ldsDNA as substrates (Supplemental Fig. S1; West et al. 1982; West and Howard-Flanders 1984). The directionality of $\mathrm{BM}$ mediated by RecA is $5^{\prime}$ to $3^{\prime}$, relative to the ssDNA gap onto which RecA binds first to form the nucleoprotein filament (Cox 2007). Thereby, it is not applicable for the DSBR model because, in this case, the 3 ' end invades to form a D-loop.

Many, if not all, eukaryotes have two kinds of RecA orthologs: Rad51 and Dmc1 (Neale and Keeney 2006). Rad51 functions in both meiotic recombination and mitotic recombinational repair, whereas Dmc1 is a meiosis-specific recombinase (Bianco et al. 1998). They both require auxiliary proteins such as $\operatorname{Rad} 55-\operatorname{Rad} 57$ from the budding yeast Saccharomyces cerevisiae and Brca2 from vertebrates in order to fully function (Sung and Klein 2006). Dmc1 has been proposed to play an important role in CO production (Bishop et al. 1992; Schwacha and Kleckner 1997; Hyppa and Smith 2010). However, both Rad51 and Dmc1 show very similar biochemical properties, and no critical differences between the in vitro activities of the two recombinases have been reported so far, at least to our knowledge.

Fission yeast Schizosaccharomyces pombe also has two recombinases: Rad51 (also called Rhp51) and Dmcl. The Swi5-Sfr1 complex is an auxiliary protein for both Rad51 and Dmc1 (Akamatsu et al. 2003, 2007; Ellermeier et al. 2004). We demonstrated that Swi5-Sfr1 stimulates three-strand exchanges mediated in vitro by Rad51 and Dmc1 (Haruta et al. 2006; Kurokawa et al.
2008). We also discovered (Murayama et al. 2008) that both fission yeast and human Rad51 proteins can promote the formation and $\mathrm{BM}$ of $\mathrm{HJs}$, with a four-strand exchange assay containing gDNA and its homologous ldsDNA (Supplemental Fig. S1). One of the most important findings in that study is that the polarity of the Rad51-mediated BM strand exchange reaction is in the $3^{\prime}$-to- $5^{\prime}$ direction, which is thus perfectly compatible with current HR models invoking the $3^{\prime}$-end invasion (Murayama et al. 2008).

In the present study, we addressed the potential ability of Dmcl to promote the formation and BM of HJs in vitro. We demonstrated such an activity for fission yeast Dmcl, but, surprisingly, the polarity of BM is in the $5^{\prime}$-to- $3^{\prime}$ direction, which is opposite to that mediated by Rad51, but the same as that mediated by RecA. This is the first time a critical difference between the biochemical properties of Rad51 and Dmc1 has been observed. The possible in vivo function of Dmcl and its effect on $\mathrm{HJ}$ formation leading to $\mathrm{CO}$ production are discussed.

\section{Results}

Dmc1 promotes a DNA four-strand exchange reaction in a 5'-to-3' direction

We demonstrated previously that Rad51 promotes the formation and $\mathrm{BM}$ of HJs in a DNA strand exchange reaction in vitro (Murayama et al. 2008). In this study, we examined whether the meiosis-specific recombinase Dmcl from fission yeast also has this activity in vitro. To monitor this, we performed a four-strand exchange reaction, which was the same as used in the previous report (Fig. 1A; Murayama et al. 2008). The reaction is initiated by pairing of the ssDNA region of the gDNA with its homologous region of ldsDNA, yielding the first joint molecule (JM) intermediate, which is denoted as a $\sigma$ structure ( $\mathrm{f} / \mathrm{M})$. An $\mathrm{HJ}$ is formed when DNA strand exchange proceeds past the ssDNA-dsDNA junction of gDNA, thereby converting the interaction from an ss-ds to duplex-duplex reciprocal strand exchange (producing an $\alpha$ structure; sJM). The completion of strand exchange yields a nicked circular (NC) DNA and an ldsDNA containing an ssDNA region (tailed DNA) as two final products. As described previously (Murayama et al. 2008), this assay system can distinguish the polarity of DNA strand exchange reactions by using different types of ldsDNA (Fig. 1A). The strand exchange using PstI-linearized ldsDNA proceeds in a $5^{\prime}$-to- $3^{\prime}$ direction relative to the ssDNA region of gDNA in a reaction termed the $3^{\prime}$-end homology reaction. Conversely, the reaction proceeds in the $3^{\prime}$-to-5' direction when HindIII-linearized ldsDNA is used as a substrate (in the $5^{\prime}$-end homology reaction). We used $5^{\prime}-{ }^{32} \mathrm{P}$-labeled ldsDNAs and analyzed the products by agarose gel electrophoresis.

We first performed a time-course experiment of the Dmc1-mediated reaction (Fig. 1B). Swi5-Sfr1 complex and RPA were added to the reaction, both of which have been shown to stimulate the three-strand exchange reaction mediated by Rad51 or Dmc1 (Haruta et al. 2006; Kurokawa et al. 2008). In both 3 '-end and 5 '-end homology 
A

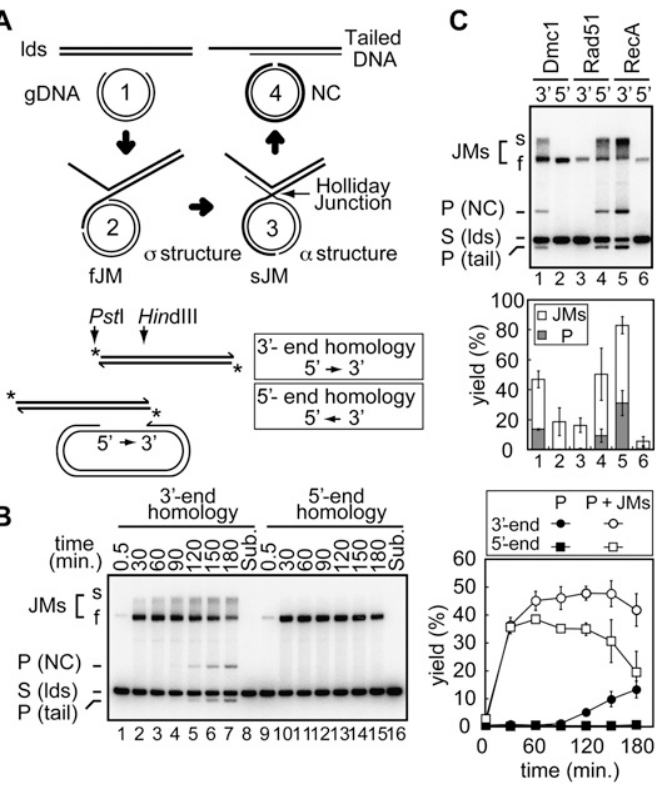

Figure 1. Dmcl promotes the four-strand exchange reaction. $(A)$ Schematic of the four-strand exchange reaction. Half arrowheads indicate $3^{\prime}$ ends of DNA. (B) The gel image shows the time-course analysis of the Dmc1-mediated four-strand exchange reaction. Large volume reactions $(50 \mu \mathrm{L})$ were performed under the standard conditions as described in the Materials and Methods. Aliquots $(6.5 \mu \mathrm{L})$ were taken at the indicated time points. The graph on the right shows the quantification of the results of the time-course analyses, in which each value and standard error was obtained from three independent experiments. (C) Determination of polarities among four-strand exchange reactions mediated by Dmc1, Rad51, and RecA. Standard fourstrand exchange was performed as described in the Materials and Methods. The incubation times were $3 \mathrm{~h}$ for Dmc1, $4 \mathrm{~h}$ for Rad51, and $40 \mathrm{~min}$ for RecA. (3') $3^{\prime}$-end reaction; (5') $5^{\prime}$-end homology reaction. The quantification of results of three independent experiments is presented in the bottom graph. (S) Substrate (ldsDNA); (P) products (NC and tailed DNA); (s) sJM, $\alpha$ structure; (f) fJM, $\sigma$ structure .

reactions,similar high amounts of JMs-especially fJMs corresponding to the $\sigma$ structure-were formed within 30 min after reaction initiation (Fig. 1B). At later time points, more slowly migrating sJMs ( $\alpha$ structure) were observed in the $3^{\prime}$-end homology reaction. The total amount of JMs in the $3^{\prime}$-end homology reaction gradually decreased, which was followed by the formation of the final products: NC and tailed DNA. The final products began to be observed $120 \mathrm{~min}$ after the reaction was initiated, and they increased in a time-dependent manner. In contrast, the $5^{\prime}$-end homology reaction did not produce any detectable sJMs or the final products, at least within 180 min. The amounts of fjMs gradually decreased in the $5^{\prime}$-end homology reaction $120 \mathrm{~min}$ after it was initiated. The reverse reaction might occur at later time points.

We then confirmed the directionality. The control experiment shown in Figure 1C demonstrates that Rad51 can produce sJMs and the two final products only in the 5 '-end homology reaction, whereas Escherichia coli RecA can produce them only in the $3^{\prime}$-end homology reaction, which is consistent with our previous study (Murayama et al. 2008). We therefore conclude that Dmcl and RecA have a preferential polarity in the four-strand reaction that is opposite to that of Rad51, which migrates in the 5'-to-3' direction relative to ssDNA in gDNA.

\section{Heterologous sequence impedes four-strand exchange mediated by Dmc1}

The time-course analysis described above suggests that final products are generated via the formation of JMs (Fig. 1A). If so, a heterologous sequence should prevent fourstrand exchange because reciprocal exchange leading to de novo complementary pairing cannot be achieved. To test this, we performed a four-strand exchange reaction using an ldsDNA containing a heterologous sequence $(0.67 \mathrm{~kb})$ at a site distal from the initial paring region (lds + het DNA) (Fig. 2A). No detectable levels of the two final products were observed in the presence of Dmcl, although sJMs accumulated in the $3^{\prime}$-end homology reaction (Fig. 2B). As controls, we analyzed four-strand exchange reactions mediated by Rad51 and RecA. In agreement with previous reports (Hahn et al. 1988; Murayama et al. 2008), neither Rad51 nor RecA produced the predicted final products, and sJMs accumulated in the $5^{\prime}$-end homology reactions of Rad51 and the 3'-end homology reaction of RecA. These results clearly indicate that an internal heterologous sequence in an ldsDNA impedes the four-strand exchange, and thus we conclude that the final products are generated via the formation of JMs in Dmc1-mediated reactions.
A

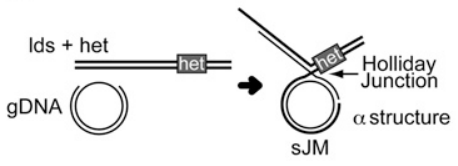

C

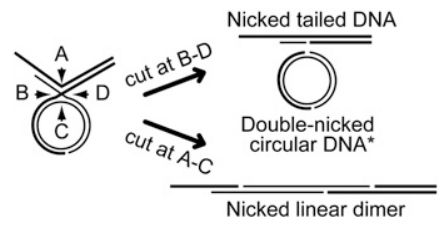

B

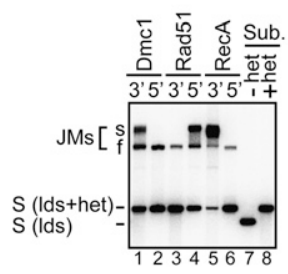

D

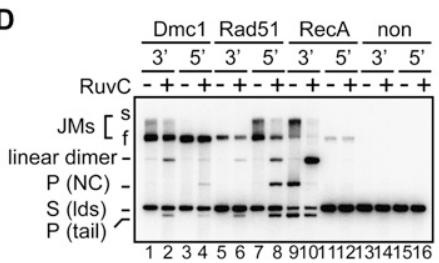

Figure 2. Formation of the $\mathrm{HJ}$ in the four-strand exchange reaction mediated by Dmc1. (A) Schematic of the four-strand exchange reaction using ldsDNA bearing a heterologous insertion (lds + het). The insertion sequence prevents $\mathrm{BM}$ of the HJ, resulting in the accumulation of $\alpha$ structures. $(B)$ Four-strand exchange reactions using lds + het mediated by Dmc1, Rad51, and RecA were performed as in Figure 1C. (Sub) Substrate ldsDNAs with heterologous insertion (+het) or not (-het). (C) Schematic of resolution of the $\alpha$ structure by RuvC. $(D)$ Four-strand exchange reactions were performed as in the presence or absence of RuvC. Note that the doublenicked circle is not detected in the $3^{\prime}$-end homology reaction, since ldsDNA was labeled with ${ }^{32} \mathrm{P}$ only at the $5^{\prime}$ end (see details in Supplemental Fig. S2). 
RuvC resolves the $\alpha$ structure formed during the four-strand exchange mediated by Dmc1

We next examined whether the JMs contained an $\alpha$ structure that corresponded to an $\mathrm{HJ}$ intermediate. To this end, we used an E. coli RuvC endonuclease that resolves HJs by introducing symmetric nicks specifically at the junction (Shinagawa and Iwasaki 1996). As shown in Figure 2C, RuvC-dependent cleavage of an $\alpha$ structure yields two sets of products: The A-C cleavage generates a nicked linear dimer duplex DNA that is twice the length of the original ldsDNA substrate, and the B-D cleavage generates a double-NC DNA and a nicked tailed DNA. Under conditions in which the four-strand reaction is not completed, the appearance of the two bands corresponding to linear dimer and tailed DNA by RuvC cleavage is an indicator of $\mathrm{HJ}$ formation during the 3 '-end homology reaction. On the other hand, the appearance of the three bands corresponding to linear dimer, NC, and tailed DNA is an indicator of the $5^{\prime}$-end homology reaction when the four-strand reaction is not completed (see Supplemental Fig. S2). To support RuvC cleavage in this assay, the concentration of free $\mathrm{Mg}^{2+}$ was adjusted to $5 \mathrm{mM}$, which was slightly higher than the optimal concentration (1.5 $\mathrm{mM}$ ) for the four-strand exchange mediated by Dmc1 (Shah et al. 1994; Takahagi et al. 1994; see below). Under this condition, JMs were produced, although the two final products were almost invisible (Fig. 2D; see below).

Based on this experimental rationale, RuvC was added to the ongoing four-strand exchange reaction. This resulted in a decrease in the amount of JMs formed during the Dmcl-mediated 3'-end homology reaction and the production of linear dimer and nicked tailed DNA after the addition of RuvC (Fig. 2D, lanes 1,2). Conversely, JMs formed during the Dmc1-mediated 5'-end homology reaction were little affected and very low levels of cleavage products were observed (Fig. 2D, lanes 3,4). Thus, we concluded that the JMs formed by Dmc1-mediated fourstrand exchange reaction contained an $\alpha$ structure that corresponded to an $\mathrm{HJ}$ intermediate. Higher amounts of RuvC cleavage products from the 3 '-end homology reaction than from the $5^{\prime}$-end homology reaction agree with the 5'-to-3'polarity of Dmc1-mediated BM, as described above. Yet, detection of RuvC cleavage products from the 5 '-end homology reaction, despite their low amounts, also suggests a potential for the $3^{\prime}$-to-5' BM mediated by Dmc1. Therefore, directionality of BM mediated by Dmcl is not as strict as that mediated by RecA (see below).

Control experiments for the RecA-mediated reactions showed that only the 3 '-end homology reaction produced RuvC cleavage products (Fig. 2D, lanes 9-12). In contrast, the $5^{\prime}$-end homology reaction by Rad51 produced RuvC cleavage products, while the $3^{\prime}$-end homology reaction by Rad51 produced very low levels of RuvC cleavage products (Fig. 2D, lanes 5-8). These results are consistent with previous reports (Dunderdale et al. 1991; Murayama et al. 2008).

Similar results were also observed in the four-strand exchange reactions using lds + het DNA (Supplemental Fig. S3A), in which BM of HJs was stalled by the heterol- ogous sequence, as described in Figure 2A. To rule out the possibility that a suboptimal concentration $(5 \mathrm{mM})$ of $\mathrm{Mg}^{2+}$ for Dmcl-mediated four-strand exchange might produce an artifact, we also performed the four-strand exchange reaction at the optimal concentration of $\mathrm{Mg}^{2+}$ for Dmc1 $(1.5 \mathrm{mM})$ and then increased the concentration of $\mathrm{Mg}^{2+}$ to the level optimal for RuvC cleavage (15 mM) (Supplemental Fig. S3B). The result was essentially the same as that shown in Figure $2 \mathrm{D}$ and Supplemental Figure S3A. Taken together, these results clearly indicate that four-strand exchange in the 3 '-end homology reaction mediated by Dmcl produces an $\alpha$ structure as an intermediate. Thus, we conclude that Dmcl promotes formation of $\mathrm{HJs}$ by a DNA pairing reaction initiating from homologous ssDNA, followed by BM into the dsDNA region.

\section{RPA/SSB, but not the Swi5-Sfr1 complex, stimulates Dmc1-mediated four-strand exchange}

We next examined the effects of two auxiliary factors: the Swi5-Sfr1 protein complex and RPA (Fig. 3). For this, we set up first the optimal concentration of Dmcl used at $5 \mu \mathrm{M}$ (Supplemental Fig. S4A). Complete reactions containing Dmc1 and the two auxiliary factors yielded $\sim 50 \%$ of JM plus the final products in $3^{\prime}$-end homology and $\sim 10 \%$ in $5^{\prime}$-end homology reactions, respectively (Fig. $3 \mathrm{~A}, \mathrm{~B}$, lanes 2). Dmc1 alone produced very small amounts of fJM in 3 '-end and 5 '-end homology reactions (Fig. 3A,B, lanes 5). When Swi5-Sfr1 was added together with Dmc1, fJM production in the 3 '-end and 5 '-end homology reactions was only slightly increased and sJMs and final products were not observed (Fig. 3A,B, lanes 3). In contrast, when RPA was added together with Dmc1,

A 3'-end homology
Dmc1 -++++--+++-++-
Swi5-Sfr1 -++--++-+-++-+ Swis-Sfr1 - ++--++-+-+-++-+-
RPA/SSB - R-R-R-RSSSSHHHH

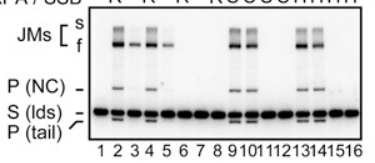

B

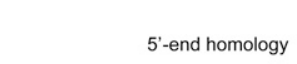
Dmc1 -++++--++-++--

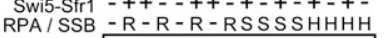
$\mathrm{RPA} / \mathrm{SSB}-\mathrm{R}-\mathrm{R}-\mathrm{R}-\mathrm{RSSSSHHHH}$
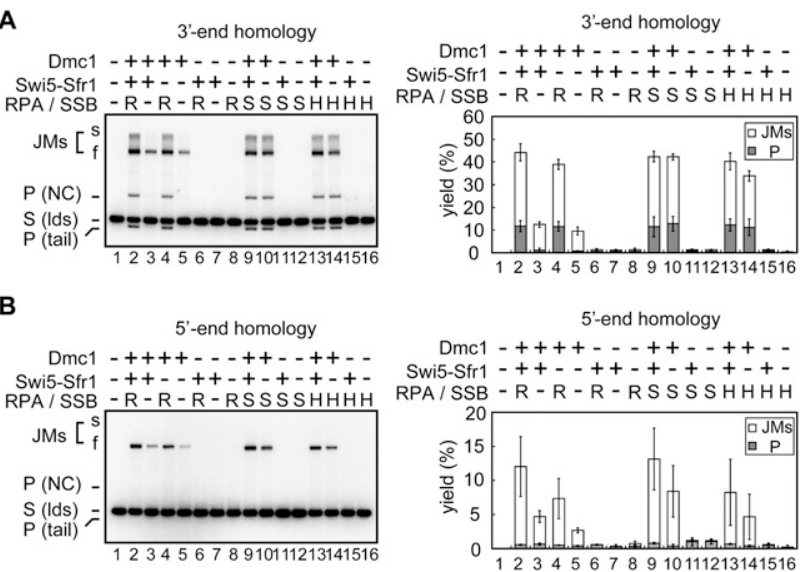

5'-end homology Dmc1 - ++++- - + + - - + + - Swi5-Sfr1 - + + - - + +- + - + - + - + RPA / SSB - R - R - R - R S S S S H H H H

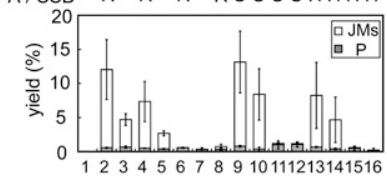

Figure 3. Effects of Swi5-Sfr1 and ssDNA-binding proteins (SSB and RPA) on the Dmc1-mediated four-strand exchange reaction. All reactions were performed with the indicated proteins (Dmc1, $5 \mu \mathrm{M}$; Swi5-Sfr1, $0.25 \mu \mathrm{M}$; RPA and human RPA, $0.5 \mu \mathrm{M}$; SSB, $0.75 \mu \mathrm{M}$ ) for $3 \mathrm{~h}$. The gel images show 3 '-end and $5^{\prime}$-end homology reactions in $A$ and $B$, respectively. The graphs on the right show the quantification of the results obtained from three independent experiments. (R) fission yeast RPA; (S) E. coli $\mathrm{SSB}_{;}(\mathrm{H})$ human RPA. Note that $Y$-axes in $A$ and $B$ are $60 \%$ and $20 \%$ of maximum, respectively. 
there was a remarkable increase in the amount of JMs and final product in the 3 '-end homology reaction (Fig. $3 \mathrm{~A}$, lane 4). This result clearly indicates that RPA, rather than the Swi5-Sfr1 complex, stimulates the 3 '-end homology reaction, which is surprising because the Swi5-Sfr1 complex is an absolute component for the three-strand exchange reaction with plasmid-sized DNAs as substrates (Haruta et al. 2006).

We next tested whether the stimulatory effect of RPA was concentration-dependent by titrating different amounts of RPA into the reactions (Supplemental Fig. S4B). RPA stimulated both 3 '-end and 5 '-end homology reactions in a protein concentration-dependent manner. The reactions were saturated at a lower amount of RPA relative to Dmc1 (approximately one-twentieth to one-tenth) in the 3 '-end homology reaction, whereas higher amounts of RPA slightly inhibited fJM formation in the $5^{\prime}$-end homology reaction.

We also found that SSB, an E. coli ssDNA-binding protein, or human RPA could substitute for fission yeast RPA in the 3 '-end homology reaction that leads to the sJM and final products (Fig. 3A, lanes 9-16). This was confirmed by time-course experiments; both bacterial SSB and human RPA, as well as fission yeast RPA, supported the Dmcl-mediated 3 '-end homology reaction four-strand exchange regardless of the presence or the absence of Swi5-Sfr1 (Supplemental Fig. S5).

Because the four-strand exchange in the 3 '-end homology reaction progressed efficiently even in the absence of the Swi5-Sfr1 complex (Fig. 3), we further examined the effect of varying the amount of the Swi5-Sfr1 complex on four-strand exchange (Supplemental Fig. S4C). Lower amounts of Swi5-Sfr1 compared with Dmc1 (approximately one-twentieth to one-tenth of Dmc1) had no effect on the final product formation, and higher amounts inhibited it in the 3 '-end homology reactions. In addition, increasing amounts of Swi5-Sfr1 resulted in increased formation of fJMs in both $3{ }^{\prime}$-end and 5 '-end homology reactions. These differences in the concentration dependency of Swi5-Sfr1 on the reactions suggest that fJM formation and the subsequent steps proceed via mechanistically different processes of Dmc1.

\section{Optimal $\mathrm{Mg}^{2+}$ concentrations are different in three-strand and four-strand exchange reactions}

The optimal $\mathrm{Mg}^{2+}$ concentration was determined (Supplemental Fig. S6). Only a narrow range of $\mathrm{Mg}^{2+}$ concentrations $(0.5-2.5 \mathrm{mM}$; optimum, $1.5 \mathrm{mM})$ supported final product formation in the 3 '-end homology reaction of fourstand exchange (Supplemental Fig. S6A). A wider range of $\mathrm{Mg}^{2+}$ concentrations (5-30 mM; optimum, $20 \mathrm{mM}$ ) supported fJM formation. Additionally, efficient three-strand exchange occurred over a wider range of $\mathrm{Mg}^{2+}$ concentrations (1-10 mM) than four-strand exchange (0.5-2.5 mM). The optimal concentration for three-strand exchange was $\sim 5 \mathrm{mM}$ (Supplemental Fig. S6B). Again, the different $\mathrm{Mg}^{2+}$ requirements suggest that three-strand and fourstrand exchange reactions are mediated by distinct mechanisms.

\section{ATP hydrolysis is essential for the BM of HJs}

ATP hydrolysis is essential for the BM of HJs mediated by Rad51 and RecA (Cox 2007; Murayama et al. 2008). To examine whether this is also true for Dmc1-mediated fourstrand exchange reaction, we performed reactions containing various adenosine nucleotide derivatives (Fig. 4A). As expected, no detectable JMs or final products were observed in either $3^{\prime}$-end or $5^{\prime}$-end homology reactions without an adenosine nucleotide (Fig. 4A, lanes 1,8) or with ADP (Fig. 4A, lanes 4,11). An ATP regeneration system was not absolutely required (Fig. 4A, lanes 3,10). A nonhydrolyzable ATP analog, ATP- $\gamma-S$, did not support JM formation or final product production (Fig. 4A, lanes 6,13), whereas another nonhydrolyzable ATP analog, AMP-PNP, supported substantial fJM formation in the $3^{\prime}$-end and 5' -end homology reactions. However, AMPPNP did not support sJM or final product formation (Fig. 4A, lanes 5,12). Because strand exchange over the ssDNA-dsDNA junction of gDNA converts fJMs to sJMs and BM in sJMs yields the final NC and tailed DNA products, we propose that the hydrolysis of ATP is required for the reciprocal exchange of two duplex DNAs, as observed previously for Rad51-mediated four-strand exchange (Murayama et al. 2008). To test this hypothesis,

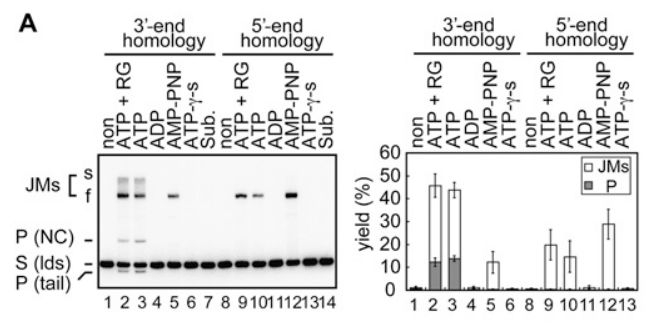

B

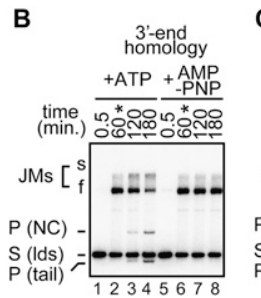

C
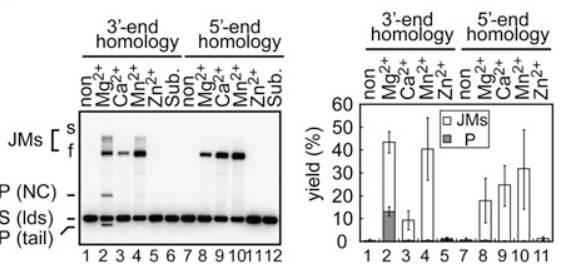

Figure 4. ATP hydrolysis is essential for BM. (A) Dmc1mediated strand exchange reactions were performed in the presence of the indicated adenosine nucleotide species $(2 \mathrm{mM})$ in the absence of an ATP regeneration system. (+RG) Addition of the ATP regeneration system. The graph on the right shows quantification of the results obtained from three independent experiments. $(B)$ AMP-PNP inhibits the BM of HJs mediated by Dmcl. The 3 '-end homology reactions $(50 \mu \mathrm{L})$ were initiated in the presence of $1 \mathrm{mM}$ ATP. At the 60-min time point (indicated by asterisks), equal amounts (1 mM) ATP (lanes 1-4) or AMPPNP (lanes 5-8) were added to the mixtures. Aliquots $(6.5 \mu \mathrm{L})$ were taken at the indicated times and analyzed by agarose gel electrophoresis. $(C)$ Effects of various divalent metal ions on the Dmc1-mediated four-strand exchange reactions. Reactions were performed in the presence of the indicated divalent ions (1.5 $\mathrm{mM}$ each). (non) Absence of a divalent ion. The quantification of results obtained from three independent experiments is shown in the graph on the right. 
a chase experiment was performed in which the $3^{\prime}$-end homology reaction was first initiated with ATP and then supplemented with an equal amount of AMP-PNP $1 \mathrm{~h}$ after initiation of the reaction (Fig. 4B). The result clearly showed that AMP-PNP inhibited the formation of sJMs and the two final products. Therefore, we conclude that the binding per se of ATP or AMP-PNP is sufficient for fJM formation from three-strand exchange, but that hydrolysis of ATP is required for the formation of HJs and their BM in the four-strand exchange reaction. The same conclusion has been reached for four-strand exchange reactions mediated by RecA and Rad51 (Kim et al. 1992; Shan et al. 1996; Murayama et al. 2008).

\section{$\mathrm{Ca}^{2+}$ does not support formation and BM of HJs} in the four-strand exchange reaction

$\mathrm{Ca}^{2+}$ stimulates D-loop formation and DNA strand exchange by human Rad51 and Dmc1 (Bugreev and Mazin 2004; Bugreev et al. 2005), and also stimulates D-loop formation by fission yeast Dmc1 (Ploquin et al. 2007). It has been proposed that $\mathrm{Ca}^{2+}$ stimulates these reactions by converting recombinases to their active ATP-bound forms through the inhibition of ATP hydrolysis (Bugreev and Mazin 2004). We examined the effects of $\mathrm{Ca}^{2+}$ on fourstrand exchange by Dmcl and found that, although $\mathrm{Ca}^{2+}$ supported fJM formation, it inhibited the formation of sJMs and the final recombination products. This finding is consistent with the requirement for ATP hydrolysis in the four-strand exchange reaction. Indeed, $\mathrm{Ca}^{2+}$ did not support the ssDNA-dependent ATPase activity of Dmc1 (Supplemental Fig. S7).

$\mathrm{Mn}^{2+}$ supports the ATPase activity and JM formation by Dmc1, but not the final product formation of the four-strand exchange

We examined the effects of divalent metal ions other than $\mathrm{Mg}^{2+}$ or $\mathrm{Ca}^{2+}$ in the Dmcl-mediated four-strand exchange reaction (Fig. 4C). Neither JMs nor final products were detected in the absence of divalent metal cations or in the presence of $\mathrm{Zn}^{2+}$ (Fig. 4C, lanes 1,5-7,11). Interestingly, $\mathrm{Mn}^{2+}$ substantially supported the formation of both fjMs and sJMs, whereas only low levels of the final products were detected. Like $\mathrm{Mg}^{2+}, \mathrm{Mn}^{2+}$ supported the ssDNAdependent ATPase activity of Dmc1 (Supplemental Fig. S7), which is consistent with the notion that BM of HJs in the four-strand exchange, but not the three-strand exchange, requires efficient ATP hydrolysis by the recombinase. In addition, this result also suggests that ATP hydrolysis by itself is not sufficient to complete the reaction. The ATP hydrolysis supported by $\mathrm{Mg}^{2+}$ is required to facilitate an efficient BM of JMs, which may involve a proper structural change of Dmcl that is coupled with the ATPase cycle.

The polarity of Dmc1-mediated strand exchange reactions

We next examined whether the polarity of BM in the fourstrand exchange reaction is due to the intrinsic strand exchange mode of Dmc1. For this, we first adopted a three-strand exchange reaction using a previously developed strand exchange assay (Murayama et al. 2008), as shown in Figure 5A. In a $3^{\prime}$-end homology reaction, only the $3^{\prime}$ region of the transferred strand of ldsDNA is complementary (thus "homologous") to the css substrate (css2), which defined the 5'-to-3' direction. Likewise, only the $5^{\prime}$ region of the transferred strand of ldsDNA is homologous to css2 in a $5^{\prime}$-end homology reaction. In the "full homology reaction," the full length of css 1 has a sequence completely complementary to that of ldsDNA, which thus should yield an NC as a final product. We used two different sites to make ldsDNAs (site 1- and site 2-linearized ldsDNAs, respectively). We also examined the effects of the end structure of the ldsDNAs; i.e., with 3 '-overhanging or $5^{\prime}$-overhanging or blunt ends.

The results demonstrated that, in the full homology reaction, JMs and NC products were substantially formed when ldsDNA with three different end structures was used, irrespective of whether it was site 1- or site 2-linearized ldsDNA (Fig. 5B, lanes 8-13). The efficiency of strand exchange was lower in the reaction using blunt end ldsDNA than in reactions using cohesive end ldsDNAs. In contrast, robust JM formation was only observed using 3 '-overhanging ldsDNA in the 3 '-end homology reaction and $5^{\prime}$-overhanging ldsDNA in the $5^{\prime}$-end homology reaction (Fig. 5B, lanes 2-7). These results suggest that the polarity of the three-strand exchange mediated by Dmc1 depends on the end structure of the ldsDNA.

We next examined the polarity or preference of Dmc1 for ssDNA ends in strand invasion. For this, we adopted a D-loop assay using three different ssDNA substrates (Fig. 5C). In the full homology reaction, the entire sequence of the 100-mer ssDNA oligonucleotide is completely complementary to the circular dsDNA substrate. In the $3^{\prime}$-end and $5^{\prime}$-end homology reactions, the 75 nucleotides (nt) on the $3^{\prime}$ or $5^{\prime}$ end of the ssDNA oligonucleotide (100-mer) are complementary, but not the remaining $25 \mathrm{nt}$, which have a random sequence. In this assay, Swi5-Sfr1 is an essential cofactor for Dmc1 because D-loop formation was not detected in any reaction with Dmc1 alone (Fig. 5C, lanes 2,8,14) or Dmc1 and RPA (Fig. 5C, lanes 5,11,17). This result demonstrated that there were no obvious differences with respect to the three different ssDNA substrates (Fig. 5C, lanes 3,9,15). RPA also stimulated D-loop formation, but only in the presence of Swi5-Sfr1. In that case, D-loop formation was more efficient in the $5^{\prime}$-end homology reaction than in the $3^{\prime}$-end homology reaction, but the full homology reaction was the most efficient of the three reactions (Fig. 5C, lanes 6,12,18). This result clearly suggests that Dmc1-mediated D-loop formation does not have a strict polarity for strand invasion, although it shows a slight preference for $5^{\prime}$-end homology when RPA is present. We therefore conclude that three-strand exchange and D-loop formation, both of which are mediated by Dmc1, can proceed in both directions relative to the ssDNA on which Dmcl forms a presynaptic filament. 
A

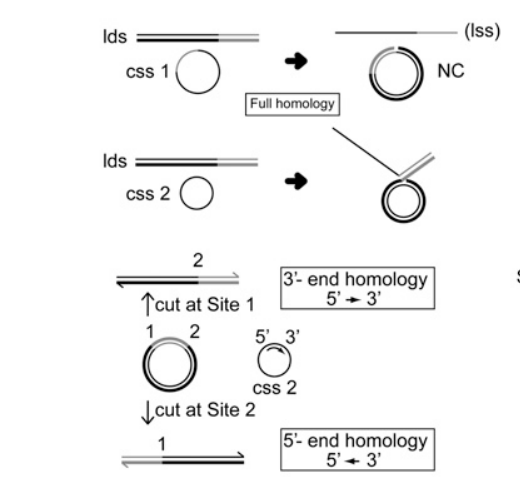

B

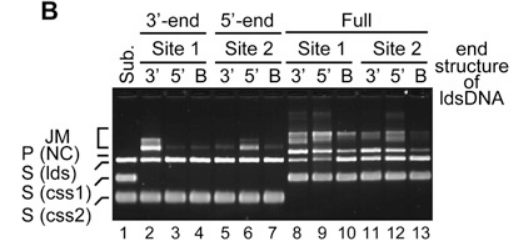

C
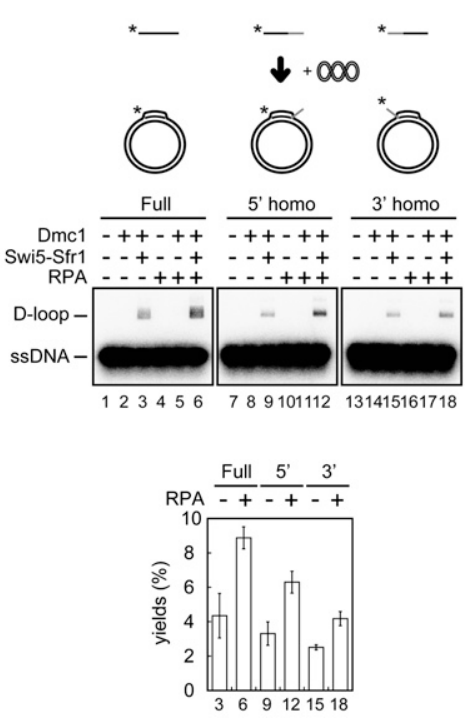

Figure 5. Polarities of three-strand exchange and D-loop formation mediated by Dmc1. (A) Schematic of the three-strand exchange reactions. css 1 is $(+)$ strand css of pSKsxAS $(4.3 \mathrm{~kb})$, and css 2 is (+) strand ssDNA of pSKsx $(2.9 \mathrm{~kb})$. pSKsxAS is a derivative of pSKsx except for the presence of an additional heterologous insertion sequence (in gray). pSKsxAS contains two restriction site clusters: Site 1 includes ApaI (3'-overhang), SalI (5'-overhang), and HincII (blunt end) sites, while site 2 includes PstI (3'-overhang), BamHI (5'-overhang), and SmaI (blunt end) sites. The combinations of css and ldsDNA yield three different types of reactions with regard to strand exchange polarity. When pSKsxAS is digested at site 1 , only the $3^{\prime}$ end of the complementary strand of the ldsDNA has homology with css2, giving a 3 '-end homology reaction. When pSKsxAS is digested at site 2 , only the $5^{\prime}$ end of the complementary strand of the ldsDNA has homology with css2, giving a $5^{\prime}$-end homology reaction. In these reactions, JMs, but not $\mathrm{NCs}$, are produced despite the completion of strand exchange. When css1, instead of css2, is used as a substrate, the entire region of ldsDNA has homology with css1, irrespective of digestion at site 1 or site 2 . This reaction is termed the full homology reaction, in which strand exchange could potentially be initiated from either end, and NCs are produced if strand exchange is completed. $(B)$ Gel image of three-strand exchange reactions mediated by Dmc1. The reactions were performed in the presence of Swi5-Sfr1 and RPA. The 3' (3'overhang), 5' (5'-overhang), and B (blunt end) above the gel image indicate the end structures of ldsDNA, which were prepared by digesting the plasmid DNA with the following restriction enzymes: for site 1, ApaI was used for the 3'-overhang, SalI was used for the 5 '-overhang, and HincII was used for the blunt end; for site 2, PstI was used for the $3^{\prime}$-overhang, BamHI was used for the 5'-overhang, and SmaI was used for the blunt end. $(C)$ Dmc1 promotes strand invasion in both directions in D-loop formation. The top panel shows a schematic of the D-loop assay. The linear ssDNA oligonucleotide (100-mer) is completely homologous to closed circular plasmid DNA (cccDNA; pBluescript SKII) in the full homology reaction. The 5' or 3' end (75-mer) of the ssDNA oligo is homologous to cccDNA in the $5^{\prime}$ or $3^{\prime}$ homology reaction, respectively. The gel images show the results of each homology reaction mediated by Dmc1 in the presence of the indicated proteins. The graph indicates the quantification of results obtained from three independent experiments.

\section{Discussion}

In this study, we demonstrated that the meiosis-specific Dmc1 recombinase from fission yeast promotes the formation and BM of HJs (Fig. 1). HJ formation is confirmed by the cleavage of the sJM formed in the fourstrand exchange by RuvC, an HJ-specific endonuclease (Fig. 2). BM polarity conferred by Dmcl is predominantly in the $5^{\prime}$-to-3' direction, relative to the ssDNA bound by Dmcl to initiate strand exchange (Figs. 1, 2). This preferential polarity is opposite to that of Rad51 (Murayama et al. 2008). To date, both Rad51 and Dmc1 recombinases have been considered to share very similar biochemical activities in vitro. Thus, the $5^{\prime}$-to-3' BM polarity of Dmc1 is the first characteristic of Dmcl that clearly distinguishes it from Rad51.

As judged by the RuvC cleavage assay, however, very small amounts of sJMs were formed in the 5'-end homology reaction, suggesting that Dmcl also promotes a low level of BM in the 3'-to-5' direction (Fig. 2D, lanes 1-4). Conversely, the same assay indicated that Rad51 promoted inefficiently BM in the 5' -to-3 direction (Fig. 2D, lanes 5-8; the result in Murayama et al. 2008), whereas the detectable levels of BM in the $3^{\prime}$-to-5' direction were not observed in the reaction mediated by RecA (Fig. 2D, lanes 9-12). Therefore, directionality of BM mediated by eukaryotic recombinases is not as strict as that mediated by RecA.
There are some critical differences between threestrand and four-strand exchanges mediated by Dmc1. The four-strand exchange (or BM) requires ATP hydrolysis, whereas ATP binding to Dmcl per se is sufficient for three-strand exchange (Fig. 4). $\mathrm{Ca}^{2+}$ supports fJM formation dependent on three-strand exchange, but not sJM formation. The optimal $\mathrm{Mg}^{2+}$ concentration for threestrand exchange is slightly higher than that for four-strand exchange (Supplemental Fig. S6). The Swi5-Sfr1 complex very efficiently stimulates three-strand exchange (Haruta et al. 2006). Conversely, the Swi5-Sfr1 complex has a very limited effect on four-strand exchange; lower amounts of Swi5-Sfr1 had no effect on the final product formation, and higher amounts inhibited it. In addition, increasing amounts of Swi5-Sfr1 resulted in increased formation of fJMs in both 3 ' -end and 5 ' -end homology reactions (Fig. 3; Supplemental Fig. S4C). Dmc1 promotes three-strand exchange in both directions, with a slight preference for the $5^{\prime}$-to- $3^{\prime}$ polarity, and this polarity is more strongly affected by the end structure of ldsDNA (Fig. 5A,B). This is in sharp contrast to the polarity observed for fourstrand exchange (Figs. 1, 2). Taken together, these results suggest that four-strand exchange is not a simple extension of three-strand exchange, but that two fundamentally different molecular mechanisms operate three-strand and four-strand exchanges, even if the same Dmcl recombinase mediates the two reactions. 
Dmc1 and Rad51 share many common features in fourstrand exchange, such as a requirement for ATP hydrolysis. Different optimal $\mathrm{Mg}^{2+}$ concentrations were found for three-strand and four-strand exchange in both Dmc1- and Rad51-mediated reactions. Three-strand exchange by both recombinases on plasmid-sized DNA substrates strictly requires the Swi5-Sfr1 complex (Haruta et al. 2006), but Swi5-Sfr1 is not essential for four-strand exchanges by either recombinase (Murayama et al. 2008; this study). Therefore, the fundamental mechanisms of four-strand exchanges mediated by Rad51 and Dmc1 may be conserved. To be precise, the four-strand exchange mediated by Rad51 is enhanced slightly by the Swi5-Sfr1 complex (Murayama et al. 2008), whereas that mediated by Dmc1 is inhibited by higher amounts of the Swi5-Sfr1 complex (this study). The most marked contrast is the BM polarities in the four-strand exchange reactions, which may reflect the different polarities of association/dissociation of each recombinase protomer onto/from ssDNA that would proceed in an ATP hydrolysis-dependent manner (Register and Griffith 1985; Bianco et al. 1998; Joo et al. 2006; Cox 2007; van Mameren et al. 2009). However, despite the fact that $\mathrm{Mn}^{2+}$ supported the ATPase activity of Dmcl, no product was observed in the presence of $\mathrm{Mn}^{2+}$ (Fig. 4C), suggesting that ATP hydrolysis by itself is not sufficient for efficient BM to produce the final products. The ATP hydrolysis supported by $\mathrm{Mg}^{2+}$ may induce a proper structural change of Dmc1 for association/dissociation, coupled with the ATPase cycle.

What is the biological significance(s) of the opposite directionalities of Dmc1 and Rad51? HJs are especially important for $\mathrm{CO}$ production in meiotic recombination, and Dmc1 is considered to be closely involved in CO formation (Bishop et al. 1992; Rockmill et al. 1995; Schwacha and Kleckner 1997; A Shinohara et al. 1997; Hyppa and Smith 2010). If true, would the $5^{\prime}$-to- $3^{\prime}$ polarity of BM be well suited to processes leading to $\mathrm{HJ}$ formation in meiotic recombination? Based on extensive studies of meiotic recombination mainly in budding yeast, the following model has been proposed for the formation of recombination intermediates (Symington 2002). After DSB generation by the Spo11 dimer, either one of two pathways proceeds to meiotic HR: One is the SDSA pathway that may not involve Dmc1, because the SDSA pathway does not produce COs or is not involved in $\mathrm{HJ}$ formation. The other major pathway is the DSBR pathway, where the canonical model involves $\mathrm{dHJ}$ formation (Szostak et al. 1983). In this pathway, Neale et al. (2005) suggest that asymmetrical nicks are introduced at DSB sites by the MRX complex. However, there is only one size class of oligonucleotides attached to Spo11, which suggests the nicking is symmetric in fission yeast (Milman et al. 2009; Rothenberg et al. 2009). Thus, the asymmetry of nicking may not be critical for DSB processing during meiosis. Although it is possible to imagine several scenarios for a role for Dmcl in the subsequent steps, and considering the requirement for Rad51 to form Dmc1 foci, the two scenarios described below (A and B) are consistent with the results of this and previous studies (Fig. 6; Sun et al. 1991; Schwacha and Kleckner 1997; Hunter and Kleckner 2001; Neale et al. 2005; Cromie et al. 2006).

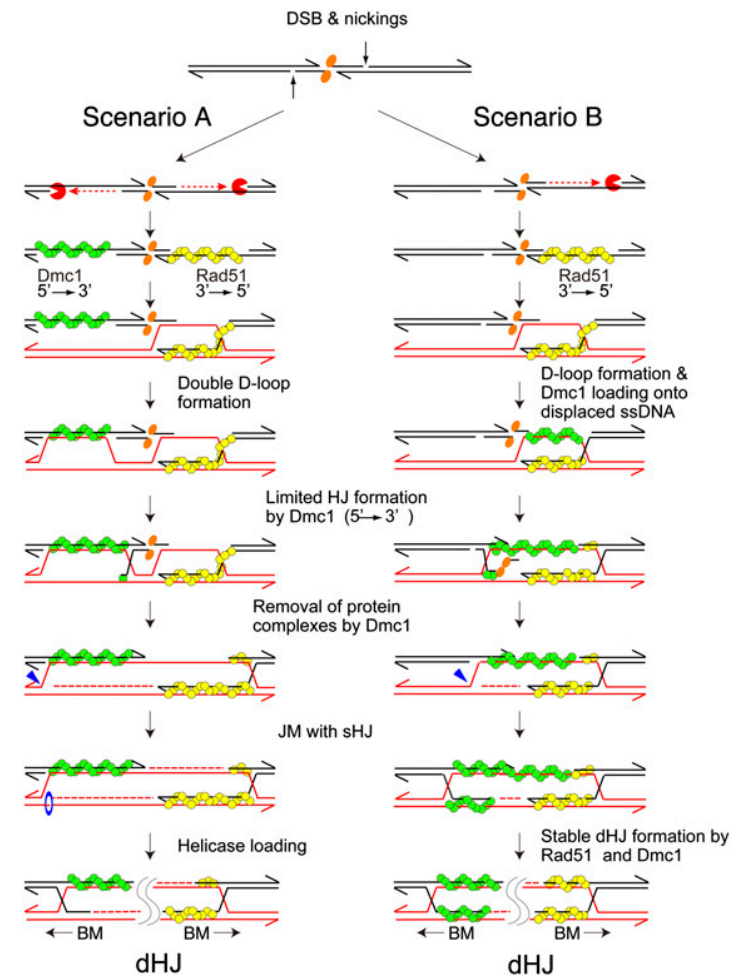

Figure 6. Possible models for the role of Dmc1-mediated fourstrand exchange reaction in HR. A Spol1 dimer (orange ellipses) creates a DSB, at the site of which the spaced nicks are introduced by the MRX (N)-Sae2 (Ctp1) (symbol not shown). Exonucleolytic resection initiates at the nicks, yielding singlestranded gaps. (Scenario $A$ ) The two single-strand gaps are used for the presynaptic filament formation of Rad51 and Dmc1, yielding two D-loops. Formation of a limited HJ by Dmc1 may lead to removal of the complexes of Spo11-ssDNA and MRX (N)-Sae2 (Ctp1). A putative helicase (a blue ring) is recruited to the end of the D-loop and forms a second HJ, creating a stable dHJ. Dashed lines in red indicate de novo DNA made by repair synthesis. If a putative nicking enzyme (blue arrowhead) introduces a nick at the end of the D-loop, a stable single HJ intermediate is created. (Scenario B) One single-strand gap is used for presynaptic filament formation of Rad51, yielding a D-loop. Dmc1 forms a presynaptic filament with the disrupted ssDNA upon D-loop formation. The extension of the Dmc1 strand exchange in the $5^{\prime}$-to- $3^{\prime}$ direction can capture the second DSB end, followed by formation of a transient HJ. The subsequent BM in the $5^{\prime}$-to-3' direction may remove the Spol1oligonucleotide and MRX (N)-Sae2 (Ctp1) complexes and reestablish another $\mathrm{HJ}$, yielding the stable $\mathrm{dHJ}$. If a putative nuclease (blue arrowhead) introduces a nick at the end of the D-loop, a stable single HJ intermediate is created.

In scenario A, exonucleolytic resection initiates at the two nicks, yielding two single-stranded gaps, but the two ends of the DSB remain associated with each other by the MRX (N)-Sae2 (Ctp1) complex (Clerici et al. 2005; for review, see Mimitou and Symington 2009). Two Spo11oligonucleotide complexes, which are stabilized by baseparing and protein-protein interactions, may also play a role in this bridging of DSB ends (Neale et al. 2005). Rad51 then forms a filament on the ssDNA gap on one side, 
leading to D-loop formation. This D-loop is converted to an $\mathrm{HJ}$, and its BM proceeds in the $3^{\prime}$-to-5' direction mediated by the intrinsic properties of Rad51 (Murayama et al. 2008). Meanwhile, Dmc1 binds to the other side of the ssDNA gap, leading to formation of a second D-loop. As strand exchange mediated by Dmcl extends in the 5'-to-3' direction into the duplex region at the DSB end, a limited HJ would be formed. Eventually, this reaction would be accompanied by the removal of the MRX (N)-Sae2 (Ctp1) complex and Spo11-oligonucleotide complexes. The resultant ssDNA gaps are sealed by repair synthesis, leading to a JM with a single $\mathrm{HJ}$ on one side. The second $\mathrm{HJ}$ is formed by a DNA helicase and/or translocase such as Tid1 (Klein 1997; M Shinohara et al. 1997; Catlett and Forsburg 2003; Bugreev et al. 2006; Nimonkar et al. 2007), which is recruited to the other side of the D-loop.

In scenario $\mathrm{B}$, exonucleolytic resection initiates at one nick, yielding a single ssDNA gap for a Rad51 filament. It forms a D-loop, followed by HJ formation, and the BM proceeds in a manner similar to that of scenario A. In scenario B, however, Dmcl is loaded onto the disrupted ssDNA upon D-loop formation. The extension of the Dmcl strand exchange in the $5^{\prime}$-to-3' direction captures the second end of the DSB, resulting in transient $\mathrm{HJ}$ formation. Subsequent BM in the 5'-to-3' direction causes the $\mathrm{HJ}$ to collapse, but continuous strand exchange reestablishes another HJ. After this reaction, both the MRX (N)-Sae2 (Ctp1) complex and Spo11-oligonucleotide complexes would be removed from the DSB ends. Finally, a stable $\mathrm{dHJ}$ is formed.

Subsequently, the distance between the two HJs is processively extended by the two BMs, each in the opposite direction, which involves, in turn, effective BM-specific motor proteins like the bacterial RuvA-RuvB complex in both scenarios A and B. Candidate proteins that may play such a role in eukaryotes are Rad54, Mer3, and Sgs1 (Rqh1 in fission yeast), although Mer3 has not been found in fission yeast.

In both scenarios, we propose a role for Dmcl in the second end capture of the DSB site. Interestingly, Rad52 has also been proposed to promote the second end capture by annealing after Rad51-mediated strand exchange (Sugiyama et al. 2006; Lao et al. 2008). Thus, it will be intriguing to examine whether Dmc1 and Rad52 function cooperatively.

Although $\mathrm{dHJs}$ are widely deemed to be a prominent intermediate in meiotic HR in many organisms, Cromie et al. (2006) have reported that single HJs are the predominant intermediate in fission yeast meiosis. They propose a model for the formation of a single $\mathrm{HJ}$ in which the branch end proximal to the $3^{\prime}$ end of the invasive ssDNA in a $\mathrm{D}$-loop is cut by a nuclease before the capture of the second end, resulting in a JM containing a single HJ. In the two scenarios mentioned above, nucleolytic nicking at the branch end can produce a JM containing a single HJ. Unlike the Cromie model, nicking occurs after second end capture in the two models proposed here, and this would seem to keep the two DSB ends together as necessary.

In budding yeast, meiotic recombination shows a bias for HR between homologs, which is more relevant for $\mathrm{CO}$ production than for HR between sister chromatids (Schwacha and Kleckner 1997). Dmcl is an important component in this pathway, at least in the SK1 strain background (Bishop et al. 1992); however, in other strain backgrounds, the Dmcl requirement is not absolute (Tsubouchi and Roeder 2003, 2006). In striking contrast, fission yeast shows a bias for HR between sister chromatids at meiotic hot spot DSBs (Cromie et al. 2006). Importantly, HR between homologs still occurs at non-hot spot DSBs in fission yeast, and Dmcl is involved in this pathway, but not in HR at hot spot DSBs (Hyppa and Smith 2010). In both yeasts, Dmc1, together with Rad51, functions in a special pathway to produce COs. The directionality of the Dmcl four-strand exchange demonstrated in this study should provide a critical clue to help elucidate the molecular mechanism of CO production.

In conclusion, we discovered a novel biochemical activity for fission yeast Dmcl that promotes four-strand exchange via the formation and BM of HJs. We showed that the three-strand and four-strand exchange reactions mediated by Dmc1 are mechanistically distinct, but that the four-strand exchange reactions mediated by Rad51 and Dmcl are similar with respect to their cofactor requirements and the need for ATP hydrolysis. The different polarities clearly distinguish these two recombinases, and may be critical elements in the pathway from HR intermediate formation to $\mathrm{CO}$ production. It will be of great interest to determine whether Dmc1 proteins from other organisms, including human Dmc1, promote fourstrand exchange with $5^{\prime}$-to-3' polarity.

\section{Materials and methods}

\section{Proteins}

Expression and purification of Dmc1, Swi5-Sfr1, Rad51, and RPA from fission yeast and RecA and RuvC from E. coli were essentially as described (Haruta et al. 2006; Kurokawa et al. 2008; Murayama et al. 2008). Human RPA was expressed in E. coli and was purified essentially as described (Henricksen et al. 1994). E. coli SSB protein was purchased from Sigma.

\section{DNA substrates}

All DNA substrates used in three-strand and four-strand exchange reactions were prepared as described (Murayama et al. 2008). A covalently closed circular plasmid DNA (cccDNA) of pBluescript SKII, which was used for D-loop analysis, was purified essentially according to the detergent lysis protocol described previously (Cunningham et al. 1980). Three 100-mer ssDNA oligonucleotides were synthesized and purified by Operon Biotechnologies. The sequences of the ssDNA oligonucleotides were as follows: IDL-2 (for full homology reaction), 5'-ACACGG AAATGTTGAATACTCATACTCTTCCTTTTTCAATATTATT GAAGCATTTATCAGGGTTATTGTCTCATGAGCGGATAC ATATTTGAATGTATTT-3'; IDL-02-75-5' het25 (for 3' homology reaction), 5'-catggaaacgaacgagagaaaacaaAATACTCATACT СTTCСTTTTTCAATATTATTGAAGCATTTATCAGGGTTA TTGTCTCATGAGCGGATACATATT-3'; IDL-02-75-3' het25 (for 5' homology reaction), 5'-AATACTCATACTCTTCCTTTT TCAATATTATTGAAGCATTTATCAGGGTTATTGTCTCAT GAGCGGATACATATTcatggaaacgaacgagagaaaacaa-3'. 
ldsDNAs used for four-strand exchange reactions and ssDNA oligonucleotides were labeled with $\left[\gamma_{-}{ }^{32} \mathrm{P}\right]$ ATP (Perkin-Elmer) and $\mathrm{T} 4$ polynucleotide kinase (TaKaRa Bio). The radiolabeled oligonucleotides were purified by gel filtration columns (MicroSpin G-25 columns, GE Healthcare).

\section{DNA strand exchange reactions}

All components used in the reactions are represented in their final concentrations. DNA concentrations are described in terms of total nucleotides. Standard reactions for Dmc1-mediated fourstrand exchange $(10 \mu \mathrm{L})$ were performed in $30 \mathrm{mM}$ Tris- $\mathrm{HCl} / \mathrm{pH}$ 7.5), $1 \mathrm{mM}$ dithiothreitol (DTT), $100 \mathrm{mM} \mathrm{NaCl}, 1.5 \mathrm{mM} \mathrm{MgCl}_{2}$, $2 \%(\mathrm{w} / \mathrm{v})$ glycerol, $2 \mathrm{mM}$ ATP, and an ATP regeneration system $/ 8$ $\mathrm{mM}$ phosphocreatine, $8 \mathrm{U} / \mathrm{mL}$ creatine kinase). After Dmcl (5 $\mu \mathrm{M})$ and Swi5-Sfr1 $(0.25 \mu \mathrm{M})$ were mixed on ice, the protein mixture was added to the reaction mixture containing gDNA $(10 \mu \mathrm{M})$ and was incubated for $10 \mathrm{~min}$ at $30^{\circ} \mathrm{C}$. RPA was added to the reaction mixture, and the mixture was incubated for an additional $10 \mathrm{~min}$ at $30^{\circ} \mathrm{C}$. After these preincubations, the fourstrand exchange reaction was initiated by the addition of ldsDNA $(8 \mu \mathrm{M})$ and incubated for $3 \mathrm{~h}$ at $37^{\circ} \mathrm{C}$. The reaction was terminated by the addition of one-fifth volume of a stop solution $(30 \mathrm{mM}$ Tris- $\mathrm{HCl}$ at $\mathrm{pH} 7.5,4.5 \%$ [w/v] SDS, $5 \mathrm{mg} / \mathrm{mL}$ proteinase K). The products were separated by $1.15 \%$ agarose gel electrophoresis with TAE buffer and analyzed as described (Murayama et al. 2008). Rad51- and RecA-mediated four-strand exchange reactions were performed as described (Murayama et al. 2008) with the following concentrations of proteins and DNA: $5 \mu \mathrm{M}$ Rad51, $0.25 \mu \mathrm{M}$ Swi5-Sfr1, $0.5 \mu \mathrm{M}$ RPA, $3.5 \mu \mathrm{M}$ RecA, $0.25 \mu \mathrm{M}$ SSB, $10 \mu \mathrm{M}$ gDNA, and $8 \mu \mathrm{M}$ ldsDNA. ATP, ADP, and ATP $\gamma$ S were purchased from Sigma-Aldrich, and AMP-PNP were purchased from Roche Applied Science.

Standard three-strand exchange reactions were performed in the same buffer conditions as for the four-strand exchange reaction except for $150 \mathrm{mM} \mathrm{NaCl}$. Dmc1 $(7.5 \mu \mathrm{M})$ and Swi5-Sfr1 $(0.75 \mu \mathrm{M})$ were mixed on ice. The protein mixture was added to the reaction mixture containing cssDNA $(15 \mu \mathrm{M})$, and the mixture was incubated for $10 \mathrm{~min}$ at $30^{\circ} \mathrm{C}$. RPA $(1.2 \mu \mathrm{M})$ was added to the reaction mixture, and the mixture was incubated for an additional $10 \mathrm{~min}$ at $30^{\circ} \mathrm{C}$. The three-strand exchange reaction was initiated by the addition of ldsDNA $(15 \mu \mathrm{M})$ and incubated for $90 \mathrm{~min}$ at $37^{\circ} \mathrm{C}$. The reaction was terminated by the addition of one-fifth volume of the stop solution and analyzed by $1 \%$ agarose gel electrophoresis with TAE buffer as described (Murayama et al. 2008).

\section{RuvC cleavage}

Four-strand exchange reactions mediated by Dmc1, Rad51, or $\operatorname{RecA}(15 \mu \mathrm{L})$ were initiated as described above except for the use of 5,10 or $15 \mathrm{mM} \mathrm{MgCl}_{2}$, respectively. At $10 \mathrm{~min}$ after the initiation of the stand exchange reaction, the reaction was divided in two aliquots ( $7 \mu \mathrm{L}$ each). RuvC ( $90 \mathrm{nM}$ final concentration) in $0.5 \mu \mathrm{L}$ of a buffer $(30 \mathrm{mM}$ Tris- $\mathrm{HCl}$ at $\mathrm{pH} 7.5,1 \mathrm{mM}$ DTT, $50 \mathrm{mM}$ $\mathrm{NaCl}, 10 \%[\mathrm{w} / \mathrm{v}]$ glycerol) or a buffer control were added to the aliquots, respectively, and they were incubated for $170 \mathrm{~min}$ for Dmc1 and Rad51 or for $30 \mathrm{~min}$ for RecA at $37^{\circ} \mathrm{C}$. The reactions were terminated by the addition of one-fifth volume of the stop solution and analyzed as described above.

\section{A D-loop assay}

A D-loop reaction $(10 \mu \mathrm{L})$ contained $30 \mathrm{mM}$ Tris- $\mathrm{HCl}(\mathrm{pH} 7.5)$, $1 \mathrm{mM} \mathrm{DTT}, 5 \mathrm{mM} \mathrm{MgCl}_{2}, 2 \%(\mathrm{w} / \mathrm{v})$ glycerol, $2 \mathrm{mM}$ ATP, and the ATP regeneration system. Dmcl $(1.5 \mu \mathrm{M})$ was mixed with a 100- mer ssDNA oligonucleotide $(3 \mu \mathrm{M})$ followed by incubation for $5 \mathrm{~min}$ at $37^{\circ} \mathrm{C}$. Swi5-Sfrl $(1.5 \mu \mathrm{M})$ or the equal volume of the Swi5-Sfr1-containing buffer was added to the mixture and further incubated for $5 \mathrm{~min}$ at $37^{\circ} \mathrm{C}$. RPA $(0.15 \mu \mathrm{M})$ or the equal amount of the RPA-containing buffer was added to the mixture. After a 5 -min incubation, the D-loop reaction was initiated by the addition of cccDNA pBluescript SKII $(90 \mu \mathrm{M})$ and was further incubated for $15 \mathrm{~min}$ at $37^{\circ} \mathrm{C}$. The reaction was terminated by the addition of one-fifth volume of stop solution. The product was separated by $1 \%$ agarose gel electrophoresis with TAE buffer. After the gel was dried on a DEAE paper, the gel image was captured using a Fuji BAS 2500 imaging plate and analyzed by Multi Gauge Analysis software (Fuji Photo Film Co.).

\section{ATPase assay}

The ATPase assay was conducted essentially as described (Haruta et al. 2006). Dmc1 $(5 \mu \mathrm{M})$ and $\operatorname{css} 1(10 \mu \mathrm{M})$ were mixed on ice in the same buffer for the four-strand exchange reaction without ATP regeneration system $(15 \mu \mathrm{L})$. The reactions were started by the addition of a mixture of $\left[\gamma^{-32} \mathrm{P}\right]$ ATP and cold ATP (final concentration, $2 \mathrm{mM}$ ) at $37^{\circ} \mathrm{C}$. Aliquots $(1.5 \mu \mathrm{L}$ ) were taken at indicated time points, and the reactions were stopped by the addition of $3 \mu \mathrm{L}$ of $0.5 \mathrm{M}$ EDTA $(\mathrm{pH}$ 8.0). Samples $(1 \mu \mathrm{L})$ were subjected to thin-layer chromatography, and the amounts of ${ }^{32} \mathrm{Pi}$ and $\left[\gamma^{-32} \mathrm{P}\right]$ ATP were determined using a PhosphorImager (BAS2500, Fuji).

\section{Acknowledgments}

We thank Professor T. Kokubo for his many discussions and continuous encouragement. We also thank Professor T. Tsurimoto for a human RPA expression plasmid, and Professor F. Uhlmann for his generous encouragement during revision. This study was supported by Grants-in-Aid for Scientific Research on Priority Innovative Areas from the Ministry of Education, Culture, Sports, Science, and Technology (MECSST) of Japan, and for Scientific Research (A) to H.I. and for Young Scientist (B) to Y.M. from the Japan Society for the Promotion of Science (JSPS), and by the Uehara Memorial Foundation to H.I.

\section{References}

Akamatsu Y, Dziadkowiec D, Ikeguchi M, Shinagawa H, Iwasaki H. 2003. Two different Swi5-containing protein complexes are involved in mating-type switching and recombination repair in fission yeast. Proc Natl Acad Sci 100: 1577015775.

Akamatsu Y, Tsutsui Y, Morishita T, Siddique MS, Kurokawa Y, Ikeguchi M, Yamao F, Arcangioli B, Iwasaki H. 2007. Fission yeast Swi5/Sfr1 and Rhp55/Rhp57 differentially regulate Rhp51-dependent recombination outcomes. EMBO $I$ 26: 1352-1362.

Allers T, Lichten M. 2001. Differential timing and control of noncrossover and crossover recombination during meiosis. Cell 106: 47-57.

Bianco PR, Tracy RB, Kowalczykowski SC. 1998. DNA strand exchange proteins: a biochemical and physical comparison. Front Biosci 3: D570-D603.

Bishop DK, Park D, Xu L, Kleckner N. 1992. DMC1: a meiosisspecific yeast homolog of $\mathrm{E}$. coli recA required for recombination, synaptonemal complex formation, and cell cycle progression. Cell 69: 439-456.

Bugreev DV, Mazin AV. 2004. $\mathrm{Ca}^{2+}$ activates human homologous recombination protein Rad51 by modulating its ATPase activity. Proc Natl Acad Sci 101: 9988-9993. 
Bugreev DV, Golub EI, Stasiak AZ, Stasiak A, Mazin AV. 2005. Activation of human meiosis-specific recombinase Dmcl by $\mathrm{Ca}^{2+}$. J Biol Chem 280: 26886-26895.

Bugreev DV, Mazina OM, Mazin AV. 2006. Rad54 protein promotes branch migration of Holliday junctions. Nature 442: 590-593.

Catlett MG, Forsburg SL. 2003. Schizosaccharomyces pombe Rdh54 (TID1) acts with Rhp54 (RAD54) to repair meiotic double-strand breaks. Mol Biol Cell 14: 4707-4720.

Clerici M, Mantiero D, Lucchini G, Longhese MP. 2005. The Saccharomyces cerevisiae Sae2 protein promotes resection and bridging of double strand break ends. I Biol Chem 280: 38631-38638.

Cox MM. 2007. Motoring along with the bacterial RecA protein. Nat Rev Mol Cell Biol 8: 127-138.

Cromie GA, Hyppa RW, Taylor AF, Zakharyevich K, Hunter N, Smith GR. 2006. Single Holliday junctions are intermediates of meiotic recombination. Cell 127: 1167-1178.

Cunningham RP, DasGupta C, Shibata T, Radding CM. 1980. Homologous pairing in genetic recombination: recA protein makes joint molecules of gapped circular DNA and closed circular DNA. Cell 20: 223-235.

Dunderdale HJ, Benson FE, Parsons CA, Sharples GJ, Lloyd RG, West SC. 1991. Formation and resolution of recombination intermediates by E. coli RecA and RuvC proteins. Nature 354: 506-510.

Ellermeier C, Schmidt H, Smith GR. 2004. Swi5 acts in meiotic DNA joint molecule formation in Schizosaccharomyces pombe. Genetics 168: 1891-1898.

Galkin VE, Wu Y, Zhang XP, Qian X, He Y, Yu X, Heyer WD, Luo Y, Egelman EH. 2006. The Rad51/RadA N-terminal domain activates nucleoprotein filament ATPase activity. Structure 14: 983-992.

Hahn TR, West S, Howard-Flanders P. 1988. RecA-mediated strand exchange reactions between duplex DNA molecules containing damaged bases, deletions, and insertions. I Biol Chem 263: 7431-7436.

Haruta N, Kurokawa Y, Murayama Y, Akamatsu Y, Unzai S, Tsutsui Y, Iwasaki H. 2006. The Swi5-Sfr1 complex stimulates Rhp51/Rad51- and Dmc1-mediated DNA strand exchange in vitro. Nat Struct Mol Biol 13: 823-830.

Henricksen LA, Umbricht CB, Wold MS. 1994. Recombinant replication protein A: expression, complex formation, and functional characterization. I Biol Chem 269: 1112111132.

Hunter N, Kleckner N. 2001. The single-end invasion: an asymmetric intermediate at the double-strand break to doubleholliday junction transition of meiotic recombination. Cell 106: $59-70$.

Hyppa RW, Smith GR. 2010. Crossover invariance determined by partner choice for meiotic DNA break repair. Cell 142: 243-255.

Joo C, McKinney SA, Nakamura M, Rasnik I, Myong S, Ha T. 2006. Real-time observation of RecA filament dynamics with single monomer resolution. Cell 126: 515-527.

Kim JI, Cox MM, Inman RB. 1992. On the role of ATP hydrolysis in RecA protein-mediated DNA strand exchange. II. Fourstrand exchanges. I Biol Chem 267: 16444-16449.

Klein HL. 1997. RDH54, a RAD54 homologue in Saccharomyces cerevisiae, is required for mitotic diploid-specific recombination and repair and for meiosis. Genetics 147: 1533-1543.

Kurokawa Y, Murayama Y, Haruta-Takahashi N, Urabe I, Iwasaki H. 2008. Reconstitution of DNA strand exchange mediated by Rhp51 recombinase and two mediators. PLOS Biol 6: e88. doi: 10.1371.journal.pbio.0060086.
Lao JP, Oh SD, Shinohara M, Shinohara A, Hunter N. 2008. Rad52 promotes postinvasion steps of meiotic double-strandbreak repair. Mol Cell 29: 517-524.

Milman N, Higuchi E, Smith GR. 2009. Meiotic DNA doublestrand break repair requires two nucleases, MRN and Ctp1, to produce a single size class of Rec12 (Spo11)-oligonucleotide complexes. Mol Cell Biol 29: 5998-6005.

Mimitou EP, Symington LS. 2009. DNA end resection: many nucleases make light work. DNA Repair (Amst) 8: 983-995.

Murayama Y, Kurokawa Y, Mayanagi K, Iwasaki H. 2008. Formation and branch migration of Holliday junctions mediated by eukaryotic recombinases. Nature 451: 1018-1021.

Neale MJ, Keeney S. 2006. Clarifying the mechanics of DNA strand exchange in meiotic recombination. Nature 442: 153158.

Neale MJ, Pan J, Keeney S. 2005. Endonucleolytic processing of covalent protein-linked DNA double-strand breaks. Nature 436: 1053-1057.

Nimonkar AV, Amitani I, Baskin RJ, Kowalczykowski SC. 2007. Single molecule imaging of Tid1/Rdh54, a Rad54 homolog that translocates on duplex DNA and can disrupt joint molecules. J Biol Chem 282: 30776-30784.

Ploquin M, Petukhova GV, Morneau D, Dery U, Bransi A, Stasiak A, Camerini-Otero RD, Masson JY. 2007. Stimulation of fission yeast and mouse Hop2-Mnd1 of the Dmc1 and Rad51 recombinases. Nucleic Acids Res 35: 2719-2733.

Register JC 3rd and Griffith J. 1985. The direction of RecA protein assembly onto single strand DNA is the same as the direction of strand assimilation during strand exchange. J Biol Chem 260: 12308-12312.

Rockmill B, Sym M, Scherthan H, Roeder GS. 1995. Roles for two RecA homologs in promoting meiotic chromosome synapsis. Genes Dev 9: 2684-2695.

Rothenberg M, Kohli J, Ludin K. 2009. Ctp1 and the MRNcomplex are required for endonucleolytic Rec 12 removal with release of a single class of oligonucleotides in fission yeast. PLoS Genet 5: e1000722. doi: 10.1371.journal.pgen.1000722.

Schwacha A, Kleckner N. 1997. Interhomolog bias during meiotic recombination: meiotic functions promote a highly differentiated interhomolog-only pathway. Cell 90: 1123-1135.

Sehorn MG, Sigurdsson S, Bussen W, Unger VM, Sung P. 2004. Human meiotic recombinase Dmc1 promotes ATP-dependent homologous DNA strand exchange. Nature 429: 433-437.

Shah R, Bennett RJ, West SC. 1994. Activation of RuvC Holliday junction resolvase in vitro. Nucleic Acids Res 22: 2490-2497.

Shan Q, Cox MM, Inman RB. 1996. DNA strand exchange promoted by RecA K72R. Two reaction phases with different Mg2+ requirements. J Biol Chem 271: 5712-5724.

Shinagawa H, Iwasaki H. 1996. Processing the Holliday junction in homologous recombination. Trends Biochem Sci 21: 107-111.

Shinohara A, Gasior S, Ogawa T, Kleckner N, Bishop DK. 1997. Saccharomyces cerevisiae recA homologues RAD51 and $\mathrm{DMCl}$ have both distinct and overlapping roles in meiotic recombination. Genes Cells 2: 615-629.

Shinohara M, Shita-Yamaguchi E, Buerstedde JM, Shinagawa H, Ogawa H, Shinohara A. 1997. Characterization of the roles of the Saccharomyces cerevisiae RAD54 gene and a homologue of RAD54, RDH54/TID1, in mitosis and meiosis. Genetics 147: 1545-1556.

Sugiyama T, Kantake N, Wu Y, Kowalczykowski SC. 2006. Rad52-mediated DNA annealing after Rad51-mediated DNA strand exchange promotes second ssDNA capture. EMBO $J$ 25: 5539-5548.

Sun H, Treco D, Szostak JW. 1991. Extensive 3'-overhanging, single-stranded DNA associated with the meiosis-specific 
double-strand breaks at the ARG4 recombination initiation site. Cell 64: 1155-1161.

Sung P, Klein H. 2006. Mechanism of homologous recombination: mediators and helicases take on regulatory functions. Nat Rev Mol Cell Biol 7: 739-750.

Symington LS. 2002. Role of RAD52 epistasis group genes in homologous recombination and double-strand break repair. Microbiol Mol Biol Rev 66: 630-670.

Szostak JW, Orr-Weaver TL, Rothstein RJ, Stahl FW. 1983. The double-strand-break repair model for recombination. Cell 33: $25-35$.

Takahagi M, Iwasaki H, Shinagawa H. 1994. Structural requirements of substrate DNA for binding to and cleavage by RuvC, a Holliday junction resolvase. J Biol Chem 269: 15132-15139.

Tsubouchi H, Roeder GS. 2003. The importance of genetic recombination for fidelity of chromosome pairing in meiosis. Dev Cell 5: 915-925.

Tsubouchi H, Roeder GS. 2006. Budding yeast Hed1 downregulates the mitotic recombination machinery when meiotic recombination is impaired. Genes Dev 20: 1766-1775.

van Mameren J, Modesti M, Kanaar R, Wyman C, Peterman EJ, Wuite GJ. 2009. Counting RAD51 proteins disassembling from nucleoprotein filaments under tension. Nature 457: 745-748.

West SC, Howard-Flanders P. 1984. Duplex-duplex interactions catalyzed by RecA protein allow strand exchanges to pass double-strand breaks in DNA. Cell 37: 683-691.

West SC, Cassuto E, Howard-Flanders P. 1982. Postreplication repair in E. coli: strand exchange reactions of gapped DNA by RecA protein. Mol Gen Genet 187: 209-217.

Yang S, Yu X, Seitz EM, Kowalczykowski SC, Egelman EH. 2001. Archaeal RadA protein binds DNA as both helical filaments and octameric rings. J Mol Biol 314: 1077-1085.

Yu X, Jacobs SA, West SC, Ogawa T, Egelman EH. 2001. Domain structure and dynamics in the helical filaments formed by RecA and Rad51 on DNA. Proc Natl Acad Sci 98: 8419-8424. 


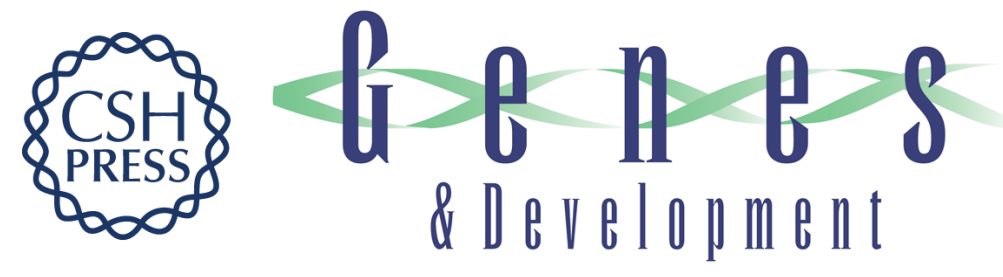

\section{The fission yeast meiosis-specific Dmc1 recombinase mediates formation and branch migration of Holliday junctions by preferentially promoting strand exchange in a direction opposite to that of Rad51}

Yasuto Murayama, Yasuhiro Tsutsui and Hiroshi Iwasaki

Genes Dev. 2011, 25:

Access the most recent version at doi:10.1101/gad.1997511

Supplemental http://genesdev.cshlp.org/content/suppl/2011/03/01/25.5.516.DC1

Material

References This article cites 56 articles, 17 of which can be accessed free at: http://genesdev.cshlp.org/content/25/5/516.full.html\#ref-list-1

License

Email Alerting Receive free email alerts when new articles cite this article - sign up in the box at the top Service right corner of the article or click here.

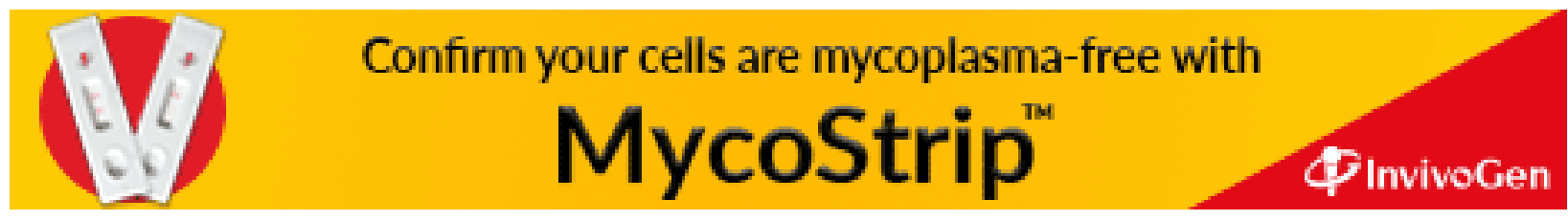

